\title{
A New Method to Prepare 3-Alkyl-2-hydroxy-1,4- naphthoquinones. Synthesis of Lapachol and Phthiocol.
}

\section{David R. da Rocha, ${ }^{a}$ Sabrina B. Ferreira, ${ }^{b}$ José Walkimar M. Carneiro, ${ }^{c}$ Wilson C. Santos, ${ }^{d}$ Vitor F. Ferreira*a}

a Universidade Federal Fluminense, Instituto de Química, Departamento de Química Orgânica, 24020-141 Niterói, Rio de Janeiro, Brazil. ' Universidade Federal do Rio de Janeiro, Instituto de Química, 27930-560, Macáe, Rio de Janeiro, Brazil. ' Universidade Federal Fluminense, Instituto de Química, Departamento de

Química Inorgânica, 24020-141 Niterói, Rio de Janeiro, Brazil. ${ }^{d}$ Universidade Federal Fluminense,

Faculdade de Farmácia, Departamento de Farmácia e Administração Farmacêutica, 24241-000, Niterói, Rio de Janeiro, Brazil.

cegvito@vm.uff.br

Keywords: Quinones, Alkylation, Lapachol synthesis

\section{INTRODUCTION}

Alkyl-1,4-naphthoquinones constitute an important group of compounds that present some types of biological activity. 3-Substituted-2-hydroxy-1,4naphthoquinones such as lapachol (1) exhibit a broad spectrum of activities. ${ }^{1}$ Lapachol $(\mathbf{1})^{2}$ is also widely used by the scientific community as a raw material for the synthesis of various bioactive derivatives and analogues, with $\beta$-lapachone (2) being one of the main lapachol derivatives.

For this reason, the isolation, structure elucidation and synthesis of 2-hydroxy-3-alkyl-1,4naphthoquinones, especially lapachol, has attracted a lot of attention.

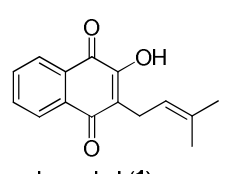

Lapachol (1)

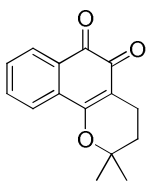

$\beta$-Lapachone (2)
Figure 1. Natural bioactive 3-substituted 1,4naphthoquinones.

\section{RESULTS AND DISCUSSION}

In this methodology, lawsone (3) was used as the starting material, and it reacted with various aldehydes via the Knoevenagel condensation, followed by reduction of the o-QM formed in situ with formic acid at high temperature (Scheme 1). Using this protocol, it was possible to prepare in moderate to excellent yields several 3-alkyl derivatives of 2hydroxy-1,4-naphthoquinone (4a-g), including lapachol (1), which are described in the Table 1.
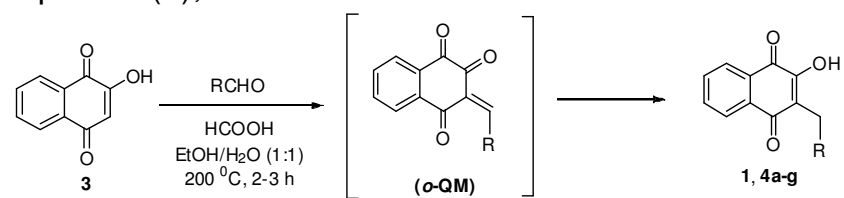

Scheme 1. Synthesis of 1,4-naphthoquinones 1 and $4 a-$ g.
The condensation and reduction reactions were performed in a closed steel reactor vessel in ethanol:water (1:1) using formic acid as reduction agent.This methodology is interesting because it could be employed to obtain 2-hydroxy-3-alkyl naphthoquinones in good yields without the possibility of formation O-alkylated by-products, one of the major problems encountered when alkylating 2-hydroxyquinones.

Table 1. Reduction of o-quinone methides to the corresponding 3-alkyl-2-hydroxy-1,4-naphthoquinones.

\begin{tabular}{|c|c|c|c|}
\hline Product & $\mathbf{R}$ & Time (h) & Yield (\%) \\
\hline $\mathbf{4 a}$ & $-\mathrm{H}$ & 3 & 89 \\
\hline $\mathbf{4 b}$ & $-p-\mathrm{NO}_{2} \mathrm{C}_{6} \mathrm{H}_{4}$ & 2 & 90 \\
\hline $\mathbf{4 c}$ & $-\mathrm{C}_{6} \mathrm{H}_{5}$ & 3 & 85 \\
\hline $\mathbf{4 d}$ & $-p-\mathrm{OCH}_{3} \mathrm{C}_{6} \mathrm{H}_{4}$ & 3 & 64 \\
\hline $\mathbf{4 e}$ & $-\mathrm{CH}\left(\mathrm{CH}_{3}\right)_{2}$ & 3 & 78 \\
\hline $\mathbf{4 f}$ & $-\mathrm{COCH}_{3}$ & 3 & 45 \\
\hline $\mathbf{4 g}$ & $-\left(\mathrm{CH}_{2}\right)_{3} \mathrm{CHO}_{1}$ & 3 & 60 \\
\hline $\mathbf{1}$ & $-\mathrm{CH}=\mathrm{C}\left(\mathrm{CH}_{3}\right)_{2}$ & 3 & 78 \\
\hline
\end{tabular}

\section{CONCLUSION}

In summary, this work describes the development of an alternative synthetic pathway for the reduction of the intermediate o-quinones methides in situ to perform selective $\mathrm{C}$-alkylation of lawsone, producing the corresponding 1,4-naphthoquinone (4a-g) in moderate to good yields, especially the lapachol (1) in $78 \%$ yield. These results indicate that this reaction method is the most efficient method to date.

\section{ACKNOWLEDGEMENTS}

CNPq, FAPERJ-PRONEX (E-26/171.512.2010), FINEP e CAPES

\section{REFERENCES}

${ }^{1}$ Santos, A. F.; Ferraz, P. A. L.; Pinto, A. V.; Pinto, M. C. F. R.; Goulart, M. O. F.; Sant'Ana, A. E. G. Int. J. Parasitol. 2000, 30, 1199.

${ }^{2}$ Hussain, H.; Krohn, K.; Ahmad, V. U.; Miana, G. A.; Greend, I. R. Arkivoc 2007, Part II, 145.

$14^{\text {th }}$ Brazilian Meeting on Organic Synthesis $-14^{\text {th }}$ BMOS - September 01-05, 2011-Brasilia, Brazil 